\title{
Green and catalytic synthesis of Dominicalure I, major component of the aggregation pheromone of Rhyzpertha dominica (Fabricius) (Coleoptera: Bostrichidae
}

\author{
Estefanía Dibello, Gustavo Seoane and Daniela Gamenara* \\ Organic Chemistry Department. Facultad de Química, Universidad de la República (UdelaR). \\ Gral. Flores 2124, 11800-Montevideo, Uruguay \\ e-mail:dgamenar@fq.edu.uy
}

\section{Supporting Information}

1.- Experimental

Description of the experiments

(S)-(+)-2-Pentanol.

rac-2-Pentanol (5.0 g, $56.8 \mathrm{mmol})$, vinyl butyrate $(6.5 \mathrm{~g}, 63.0 \mathrm{mmol})$ and lipase B from

C. antarctica -Novozym 435- (100 mg) were stirred in an orbital shaker (150 rpm) at $37^{\circ} \mathrm{C}$ for 15 minutes. The reaction was monitored by chiral gas chromatography. The enzyme was filtered and (S)-2-pentanol was separated from the $(R)$-butyl ester by column chromatography (Hexane:AcOEt 8:2). Yield 49\%, > 99\% ee. $[\alpha]_{\mathrm{D}}{ }^{25}=+13.5$ $\left(\mathrm{CHCl}_{3}, \mathrm{c}=0.5 \mathrm{~g} / 100 \mathrm{ml}\right)$.

(E)-2-Methyl-2-pentenal (2). ${ }^{[1]}$ 
Pyrrolidine (0.5 g, $7.7 \mathrm{mmol}$ ) was added to propanal (3.0 g, $51.6 \mathrm{mmol})$ in hexane (20 $\mathrm{ml})$. The reaction was stirred at room temperature for 48 hours and then $2 \mathrm{ml}$ of a $10 \%$ $\mathrm{HCl}$ solution was added, and kept at room temperature for 4 hours. The crude mixture was extracted with hexane $(3 \times 5 \mathrm{ml})$ and then washed with $10 \% \mathrm{HCl}$ to $\mathrm{pH}=7$. The combined organic layers were washed with brine $(2 \times 5 \mathrm{ml})$. The solvent was distilled under atmospheric pressure, and aldehyde $\mathbf{2}$ was obtained as a single isomer after distillation under the same conditions. Yield 95\%. ${ }^{1} \mathrm{H}$ NMR: $\delta(\mathrm{ppm})=9.40(\mathrm{~s} ; 1 \mathrm{H}), 6.49$ (tq; $\left.J_{1}=7.3 \mathrm{~Hz}, J_{2}=1.3 \mathrm{~Hz}, 1 \mathrm{H}\right), 2.37(b r \mathrm{q} ; J=7.5 \mathrm{~Hz}, 2 \mathrm{H}), 1.75(b r \mathrm{~d} ; J=0.9 \mathrm{~Hz}, 3 \mathrm{H})$, $1.12(\mathrm{t} ; J=7.6 \mathrm{~Hz}, 3 \mathrm{H}) .{ }^{13} \mathrm{C}$ NMR: $\left.\delta(\mathrm{ppm})=195.7,156.3,138.8,22.4,12.9,9.1\right) . \mathrm{GC}: \mathrm{R}_{\mathrm{t}}$ = $10.74 \min \left(\mathrm{DB} 5\right.$ column). MS (IE; $70 \mathrm{eV}: \mathrm{m} / \mathrm{z}=99\left(\mathrm{M}^{+}+1,9 \%\right), 98\left(\mathrm{M}^{+}, 92 \%\right), 97$ (25\%), 95 (10\%), 87 (8\%), 83 (44\%), 81 (10\%), 79 (10\%), 75 (10\%), 70 (35\%), 69 (58\%), 67 (18\%), 59 (11\%), 58 (9\%), 57 (100\%), 56 (10\%), 55 (54 \%), 53 (16\%), 44 (27\%), 43 (92\%), 42 (18\%), 41 (74\%), 29 (23\%).

\section{(E)-Methyl 2-methyl-2-pentenoate (3). ${ }^{[1]}$}

$\mathrm{NaCN}$ (98.0 mg, $0.36 \mathrm{mmol}), \mathrm{MnO}_{2}(690.0 \mathrm{mg}, 7.92 \mathrm{mmol})$ and acetic acid (36.7 mg, $0.6 \mathrm{mmol})$ were added to a stirred solution of $2(35.0 \mathrm{mg}, 0.36 \mathrm{mmol})$ in $3.0 \mathrm{ml}$ of $\mathrm{MeOH}$. The reaction system was equipped with an adequate device provided with a $\mathrm{KOH}$ solution in order to neutralize the exceeding $\mathrm{HCN}$ generated during the reaction. The reaction was stirred at room temperature for 16 hours, then filtered through celite and washed exhaustively with diethyl ether. The organic solvent was distilled under reduced pressure. The crude mixture was dissolved in $\mathrm{Et}_{2} \mathrm{O}(20 \mathrm{ml})$, the organic layer was washed with brine $(2 \times 5 \mathrm{ml})$, and the solvent was removed by distillation under reduced pressure. The product was purified by column chromatography Hexane:AcOEt 99:1 to 3:7. Yield: 80\%. GC: $\mathrm{R}_{\mathrm{t}}=6.47 \mathrm{~min}$ (DB5 column). MS (IE; $70 \mathrm{eV}: \mathrm{m} / \mathrm{z}=129$ 
( $\left.\mathrm{M}^{+}+1,3 \%\right), 128$ (M+3 $\left.\mathrm{M}^{+}, 36\right), 113$ (13\%), 99 (9), 97 (23\%), 96 (9\%). 95 (8\%), 85 (4\%),

81 (4\%), 69 (80\%), 68 (12\%), 67 (23\%), 65 (4\%), 59 (22\%), 55 (9\%), 54 (4\%), 53

(19\%), 52 (3\%), 51 (5\%), 50 (3\%), 45 (5\%), 43 (18\%), 42 (7\%), 40 (9\%), 39 (36\%), 38

(3\%), 32 (34\%), 31 (3\%), 29 (13\%).

(E)-(S)-(+)-1-methylbutyl 2-methyl-2-pentenoate (Dominicalure I). ${ }^{[2]}$

(S)-2-Pentanol (0.2 g, $2.4 \mathrm{mmol}$ ) and lipase B from C. antarctica (Novozym 435, 30 $\mathrm{mg}$ ) were added to a solution of $3(200 \mathrm{mg}, 1.6 \mathrm{mmol})$ in $3 \mathrm{ml}$ of hexane. The reaction mixture was stirred in an orbital shaker (150 rpm) at $37^{\circ} \mathrm{C}$ for 96 hours. The enzyme was filtered and the crude mixture was purified by column chromatography (Hexane:AcOEt 95:5). Yield: 90\%. $[\alpha]_{\mathrm{D}}{ }^{25}=+13.4\left(\mathrm{Et}_{2} \mathrm{O}, c=0.184 \mathrm{~g} / 100 \mathrm{ml}\right) .{ }^{1} \mathrm{H}$ NMR: $\delta(\mathrm{ppm})=6.72\left(\mathrm{tq} ; J_{1}=7.3 \mathrm{~Hz}, J_{2}=1.4 \mathrm{~Hz}, 1 \mathrm{H}\right), 4.95-5.02(\mathrm{~m} ; 1 \mathrm{H}), 2.18(b r \mathrm{q} ; J=7.5$ Hz, 2H), 1.82 (br d; $J=1.3 \mathrm{~Hz}, 3 \mathrm{H}), 1.61-1.66$ (m; 1H), 1.47-1.55 (m; 1H), 1.30-1.42 (m; 3H), 1.26 (d, $J=6.3,3 \mathrm{H}), 1.05$ (t; $J=7.6 \mathrm{~Hz}, 3 \mathrm{H}), 0.92(\mathrm{t} ; J=7.3 \mathrm{~Hz}, 3 \mathrm{H}) .{ }^{13} \mathrm{C}$ NMR: $\delta(p p m)=168.0,143.3,127.6,70.7,38.2,22.0,20.0,18.7,14.0,13.1,12.2 . \mathrm{GC}:$ $\mathrm{R}_{\mathrm{t}}=11.67 \mathrm{~min}$ (DB5 column). MS (IE; $70 \mathrm{eV}: \mathrm{m} / \mathrm{z}=184$ (M+1\%), 155 (5\%), 141 (1\%), 116 (5\%), 115 (88\%), 114 (10\%), 99 (4\%), 98 (8\%), 97 (100\%), 96 (3\%), 87 (6\%), 85 (2\%), 71 (11\%), 70 (43\%), 69 (50\%), 68 (4\%), 67 (9\%), 55 (18\%), 54 (4\%), 53 (9\%), 45 (8\%), 43 (51\%), 42 (13\%), 41 (63\%), 40 (7\%), 39 (18\%), 32 (31\%), 29 (14\%).

\section{1.- References}

[1] Chan, K. C.; Jewell, R. A.; Nutting, W. H.; Rapoport, H. J. Org. Chem. 1968, 33, 3382-3385. 
[2] Liu, L.-Y.; Lin, G.-Q. J. Chem. Ecol. 1990, 16, 1921-1925. 